\title{
Fairness Time-Slot Allocation and Scheduling with QoS Guarantees in Multihop WiMAX Mesh Networks
}

\author{
Chien-Yu Wu, ${ }^{1}$ Hann-Jang Ho, ${ }^{2}$ Sing-Ling Lee, ${ }^{1}$ and Liang Lung Chen ${ }^{1}$ \\ ${ }^{1}$ Institute of Computer Science and Information Engineering, National Chung Cheng University, \\ No. 168, Section 1, University Road, Min-Hsiung Township, Chia-yi County 62102, Taiwan \\ ${ }^{2}$ Department of Applied Digital Media, WuFeng University, No. 117, Section 2, Chiankuo Road, \\ Min-Hsiung Township, Chia-yi County 62153, Taiwan \\ Correspondence should be addressed to Hann-Jang Ho; hannjangho@gmail.com
}

Received 17 September 2013; Accepted 9 October 2013

Academic Editor: Teen-Hang Meen

Copyright (C) 2013 Chien-Yu Wu et al. This is an open access article distributed under the Creative Commons Attribution License, which permits unrestricted use, distribution, and reproduction in any medium, provided the original work is properly cited.

The WiMAX technology has been defined to provide high throughput over long distance communications and support the quality of service (QoS) control applied on different applications. This paper studies the fairness time-slot allocation and scheduling problem for enhancing throughput and guaranteeing QoS in multihop WiMAX mesh networks. For allocating time slots to multiple subscribe stations (SSs), fairness is a key concern. The notion of max-min fairness is applied as our metric to define the QoSbased max-min fair scheduling problem for maximizing the minimum satisfaction ratio of each SS. We formulate an integer linear programming (ILP) model to provide an optimal solution on small-scale networks. For large-scale networks, several heuristic algorithms are proposed for better running time and scalability. The performance of heuristic algorithms is compared with previous methods in the literatures. Experimental results show that the proposed algorithms are better in terms of QoS satisfaction ratio and throughput.

\section{Introduction}

In recent years, worldwide interoperability for microwave access (WiMAX), the broadband wireless access technology based on IEEE 802.16 standard [1], has received enormous attention in wireless communication networks. Based on IEEE 802.16, WiMAX system has been defined to provide high throughput over long distance and to support the quality of service (QoS) control applied on different applications. The IEEE 802.16 standard supports both the point-to-multipoint (PMP) mode and the mesh mode. In PMP mode, stations are organized as a cellular network, where subscriber stations (SSs) are directly connected to base stations (BSs). Such networks require each SS to be within the communication range of its associated BS, thus greatly limiting the coverage range of the network. In the mesh mode, the mobile stations are connected as an ad hoc network. Moreover, the mobile stations send the packets to the neighbors; the neighbors relay the received packets to the base station. Thus, it is unnecessary to have a direct connection between each mobile station and the base station.
In an IEEE 802.16 mesh network, transmissions can undergo a multihop manner. The standard specifies a centralized scheduling mechanism for the BS to manage the network. Stations will form a routing tree rooted at the BS for the communication purpose. SSs in the network will send request messages containing their traffic demands to the BS to ask for resources. The BS then uses the topology information along with SSs' requests to determine the routing tree and to allocate resources. All next generation cellular wireless systems employ orthogonal frequency division multiple access (OFDMA) based multicarrier technology. An OFDMA frame consists of time slots in the time domain and subchannels in the frequency domain. A time-slot and subchannel combination, referred to as a tile, is the minimum allocable unit.

In the wireless transmission technology, the schedule for data transmission is a very important research issue. The main purpose of the schedule includes: (1) increasing the network throughput, (2) shorting the scheduled time, (3) providing a guarantee QoS service, and (4) keeping transferring 
fairness in the network. Therefore, it is very important to have a good scheduling algorithm in the wireless transmission.

Since current wireless network systems usually use the OFDMA as communication technology, they also plan to provide various QoS services for a large number of users. The high data transfer throughput and QoS assurance have become the main goal of the wireless network [2-4]. To get more efficient bandwidth usage and to provide better QoS services to users of the wireless network, dynamic resource allocation method has been widely studied in $[2,3]$. When we consider the real-time service flows, such as VoIP and wireless multimedia communications, their quality of services (QoSs) should be satisfied. The real-time connections will periodically transmit or receive a constant amount of traffic.

Recently, several literatures discuss the scheduling scheme for multihop transmissions in WiMAX mesh networks, since the standard protocol usually does not specify any particular scheduling scheme. For example, the throughput and fairness issues have been studied in [5]. However, the previous literature does not guarantee the QoS while considering the fairness scheduling of SSs in real time. It is worth exploring how the well-known fairness schemes such as max-min, max-flow, absolute, and proportional fairness can be implemented in 802.16j networks with the QoS constraint. This paper concentrates on the time-slot allocation and scheduling for WiMAX mesh networks with OFDMA protocol. The goal of this paper is to provide various scheduling schemes which optimize both QoS and fairness of resource allocations for the WiMAX mesh networks. From the experimental results, we show that our proposed schemes are better than the previous work in terms of QoS satisfaction ratio.

This paper applies the concept of max-min fair scheduling to enhance the minimum satisfaction ratio over the WiMAX mesh networks. We propose an ILP model to solve the problem and provide heuristic methods to ensure the QoS priority of scheduling to achieve better overall QoS satisfaction ratio and throughput.

The main contributions of our work are listed below.

(1) Propose a heuristic algorithm that can optimize the max-min fair and guarantee QoS. In our proposed method, QoS requirements are a primary consideration for allocating resources to various SSs. If there are remaining resources after stratifying the QoS requirements, we will then consider the max-min fair scheduling to improve the overall throughput and minimize the satisfaction ratio. In IEEE 802.16j mesh networks, the proposed algorithm can meet the QoS requirements of various SSs in a frame and enhance the network overall QoS satisfaction ratio.

(2) Exploit spectral reuse. The spectral reuse is adopted by our heuristic algorithm to avoid the packets collisions to improve the network throughput and QoS satisfaction ratio.

(3) Construct the ILP model. We construct an integer linear programming model which constraints on the QoS requirements and max-min fairness assignments for solving the optimization problem in multihop WiMAX mesh networks.

\section{Network Model}

2.1. MMR Infrastructure. This paper focuses on the time-slot allocation and scheduling on the network system with IEEE 802.16j mobile multihop relay-based (MMR) infrastructure. In accordance with the recommendation of WiMAX standard [6], BS is the root of the tree to assist the transmission of WiMAX mesh networks. The RSs are the intermediate nodes of the tree, and the SSs are the leaf node of the tree.

We mainly focus on the scheduling between the SSs and RSs. Our goal is to find a scheduling method which can meet the QoS requirements of each SS and achieve a fair allocation of network resources. We also study how to allocate subchannels and time slots according to the bandwidth requirements of each SS in a frame. We assign the transmission schedule to maximize the minimum satisfaction ratio over all SSs to get the overall max-min fairness for the multihop relayed WiMAX mesh network.

2.2. Mesh Mode. We focus on the 802.16 mesh network which is composed by one BS and several SSs. The BS is responsible for connecting the back-end network. Each SS transfers data to neighboring SSs without the BS's agreement. The data flow away from the BS is called downlink data flow; conversely, which toward the BS is called uplink data flow. For mesh networks, most studies focus on topology design [7]. In 802.16 mesh networks, some issues are studied for supporting QoS in $[8,9]$. Shetiya and Sharma [8] studied the QoS routing problem of Central Scheduling. Hong and Pang [9] considered the multihop scheduling problem with bandwidth and delay constraints. In these researches, different approaches for establishing the routing tree of mesh networks are analyzed, and some issues of time-slot allocation are discussed.

2.3. Spectral Reuse. This paper applies spectral reuse to solve the resource allocation problem for the 802.16 mesh network. The advantage of using spectral reuse is to improve the transmission capacity and the throughput of network. Fu et al. [10] proposed an algorithm to maximize the usage of spectral reuse. Chen et al. [11] studied how to use the spectral reuse to solve the problem of resource allocation.

2.4. Interference Model. Scheduling strategies must ensure that transmissions in each time slot do not collide. There are two types of collision situations in the wireless network environment. They are called Primary Interference and Secondary Interference $[12,13]$. Primary Interference occurs in a single time slot of scheduling; the SS cannot do more than one thing. In other words, the SS can only transfer or receive data in a single time slot. Secondary Interference occurs when the originally receiver $X$ turns into the transfer, but the user $X$ is still in the range of the transfer $Y$. The user $X$ will affect the transmission of the user $Y$. There are some studies that focus on WiMAX mesh network interference problems [14, 15], Wei et al. [16] proposed an interference-aware multihop 
TABle 1: Parameters and variables table.

\begin{tabular}{|c|c|}
\hline Symbol & Description \\
\hline$G=(V, E)$ & Mesh network topology $G$ \\
\hline$V$ & The set of stations \\
\hline$E$ & The set of edges \\
\hline$T$ & The set of time slot in a frame \\
\hline$H$ & The set of subchannel in each time slot \\
\hline$h$ & The index parameter of subchannel, $\forall h \in H$ \\
\hline $\mathbb{S}$ & A set of all the SSs \\
\hline$s_{i}$ & A subscriber $\mathrm{SS} i, \forall s_{i} \in \mathbb{S}$ \\
\hline $\mathbb{R}$ & Set of all relay stations \\
\hline$r_{i}$ & A relay node $i, \forall r_{i} \in \mathbb{R}$ \\
\hline$c_{l}$ & The link capacity of each link $l, \forall l \in E$ \\
\hline $\mathrm{hp}_{i}$ & The set of hops from $s_{i}$ to BS \\
\hline $\mathrm{br}_{i}$ & The minimum bandwidth requirement of the $s_{i}$ for guaranteeing QoS \\
\hline $\mathrm{BR}_{i}$ & The maximum bandwidth requirement of the $s_{i}$ \\
\hline$\lambda_{i}$ & The actual quantity of node $i$ upload the packet, $\forall i \in \mathbb{S} \cup \mathbb{R}$ \\
\hline$\sigma_{i}$ & The actual quantity of node $i$ upload the required tile, $\forall i \in \mathbb{S} \cup \mathbb{R}$ \\
\hline$y_{i}$ & $s_{i}$ satisfaction ratio \\
\hline $\operatorname{tr}_{i}$ & The tile requirement of $s_{i}$ for transmitting packets from $s_{i}$ to BS during each hop \\
\hline TR & The set of tile requirement, $\mathrm{TR}=\left\{\operatorname{tr}_{i} \mid \forall s_{i} \in \mathbb{S}\right\}$ \\
\hline$I(\cdot)$ & The subset of nodes which will interfere with node or link transceiving \\
\hline $\bar{I}_{i}$ & The subset of nodes which will not interfere with $s_{i}^{\prime} s$ transceiving \\
\hline $\mathrm{pa}(i)$ & The parent of node $i$ on the tree topology \\
\hline $\operatorname{cd}(r)$ & The set of children node SSs or RSs of $r$ \\
\hline$f_{t, h}^{l}$ & A decision variable, which is 1 if $\operatorname{link} l$ is assigned with time slot $t$ and subchannel $h$, and 0 otherwise \\
\hline $\mathrm{OL}_{i}, \mathrm{IL}_{i}$ & The set of output and input links of node $i$, respectively \\
\hline
\end{tabular}

routing algorithm to maximize the degree of use of network bandwidth to maximize the network throughput.

2.5. Fairness Scheduling. Nowadays the related works on WiMAX mesh networks through a network of relay stations scheduling and resource allocation are concerned widely. It is common to study the fairness in many wireless networks in [17-20]. Sayenko et al. [19] studied the proportional fairness and considered the difference between frequency selections with multiuser scheduling problems. Andrews and Zhang [20] proposed a round-robin based scheduling method for IEEE 802.16 BS to ensure QoS requirements of the SS in uplink (UL) and downlink (DL) can be met. But the network bandwidth usage efficiently and bandwidth requirements were not considered in $[19,20]$.

In this paper, we propose heuristic scheduling algorithms that identify each SS to maximize the minimum satisfaction ratio in the network and, meanwhile, to meet the bandwidth requirements for each SS. We compare the QoS satisfaction ratio, throughput, and min satisfaction ratio with the previous method proposed in [21]. Experimental results show that our method is better in terms of QoS satisfaction ratio.

\section{QoS-Based Max-Min Fair Scheduling}

3.1. Problem Definition. Based on the WiMAX standard [1], a tree network topology $G=(V, E)$ is given. For readability, the following parameters and variables are listed in Table 1. A BS as the root, a set of subscriber users $\mathbb{S}=\left\{s_{1}, s_{2}, \ldots, s_{n}\right\}$ as the leaf nodes, and a set of relay stations $\mathbb{R}=\left\{r_{1}, r_{2}, \ldots, r_{m}\right\}$ as the intermediate nodes. Let the parameter $c_{l}$ be the link capacity of each link $l, \forall l \in E$. Let the parameter br $_{i}$ be the minimum bandwidth requirements of each SS $s_{i}$ during a frame. Let the parameter $\mathrm{BR}_{i}$ be the maximum bandwidth requirements of each SS $s_{i}$ during a frame. The problem is limited to the following restrictions:

(1) no spectral reuse for any pair of links which interfere with each other;

(2) an RS cannot transmit and receive data at the same time;

(3) the total number of data delivered by an RS to BS during a frame must be equal to the number of data received from its children node during one frame;

(4) must satisfy the minimum bandwidth requirements of each SS to guarantee QoS. 
Therefore, the multihop fair scheduling with QoS control problem is defined as to find a way to schedule the subchannel-time slot (tile) for a scheduling frame. After the tile scheduling, the minimum satisfaction ratio $y_{i}$ of each $s_{i}$ will be maximized, and the bandwidth requirements of each $s_{i}$ will be satisfied to guarantee QoS.

3.2. Integer Linear Programming for the Problem. In [22], it was proved that scheduling with channel capacity is NP-hard. Therefore, our scheduling problem with time-varying channel will be NP-hard. To find an optimal solution, we provide an Integer Linear Programming (ILP) for this problem.

For each node $i \in \mathbb{S} \cup R$, pa $(i)$ denotes the parent of node $i$ on the tree topology. For each $\mathrm{RS} r \in \mathbb{R}$, the parameter $\operatorname{cd}(r)$ is used to represent the set of children node (SSs or RSs) of $r$. For each node $i$ in tree network $G$, the variable $f_{t, h}^{l}$ denotes that whether the link $l=(i, j)$ is assigned with time-slot $t$ and subchannel $h$. We refer to the method $[23,24]$ to verify whether there are interferences between these two edges. $I(l)$ represents the interference link set of link $l$ :

$$
\begin{gathered}
\text { Maximize } y \\
\sum_{l^{\prime} \in I(l)} f_{t, h}^{l^{\prime}}+f_{t, h}^{l} \leq 1, \quad \forall h \in H, \quad \forall t \in T, \quad \forall l \in \mathrm{OL}_{i}, \\
\sum_{\forall h \in H} \sum_{\forall l \in \mathrm{OL}_{r}} f_{t, h}^{l}+\sum_{\forall h \in H} \sum_{\forall l \in \mathrm{IL}_{r}} f_{t, h}^{l} \leq 1, \quad \forall t \in \mathbb{S} \cup \mathbb{R}, \\
\sum_{\forall l \in \mathrm{IL}_{r}}\left(\sum_{\forall h \in H} \sum_{\forall t \in T} f_{t, h}^{l}\right) \times \mathcal{c}_{l} \\
\leq \sum_{\forall l \in \mathrm{OL}_{r}}\left(\sum_{\forall h \in H} \sum_{\forall t \in T} f_{t, h}^{l}\right) \times c_{l}, \quad \forall r \in \mathbb{R}, \\
\sum_{\forall l \in \mathrm{OL}_{i}}\left(\sum_{\forall h \in H} \sum_{\forall t \in T} f_{t, h}^{l}\right) \times c_{l} \geq \mathrm{br} r_{i}, \quad \forall i \in \mathbb{S}, \\
y \leq y_{i}, \quad \forall i \in \mathbb{S}, \\
\text { where } y_{i}=\frac{1}{\mathrm{BR}} \sum_{i \forall l \in \mathrm{OL} \mathrm{L}_{i}}\left(\sum_{\forall h \in H \forall t \in T} \sum_{t, h}^{l}=\{0,1\}, \quad \forall t \in T, \forall h \in H, \quad \forall l \in E .\right.
\end{gathered}
$$

The objective function of QoS-based max-min fair scheduling problem is shown as (1). The goal is to maximize the minimum satisfaction ratio $y$. The satisfaction ratio $y_{i}$ is calculated by (7). In (7), the parameter $\mathrm{BR}_{i}$ is the maximum bandwidth requirement of $s_{i}$, the parameter $c_{l}$ is the capacity of link $l$, and the decision variables $f_{t, h}^{l}$ are defined in (8). If the time-slot $(t, h)$ is allocated to $\operatorname{link} l$, the value of decision variable $f_{t, h}^{l}$ is 1 . Otherwise, the value of decision variable $f_{t, h}^{l}$ is 0. The QoS-based max-min fair scheduling problem has four constraints as follows.

(i) The spectral reuse constraints are shown as (2). For all link $l$ in $\mathrm{OL}_{i}$, a tile can be used no more than once in each pair of interference links $I(l)$. Where the $\mathrm{OL}_{i}$ is the set of output links of all nodes $i \in \mathbb{S} \cup \mathbb{R}$.

(ii) The single transceiver constraints are shown as (3). For all time-slot $t$ in $T$, each RS $r$ in $R$ cannot transmit and receive data in the same time slot.

(iii) The flow constraints are shown as (4). All data that are accepted by RS $r$ in a frame will be sent out in the same frame. Where the parameters $\mathrm{OL}_{r}$ and $\mathrm{IL}_{r}$ are the set of output and input links of RS $r$.

(iv) The minimum bandwidth requirement constraints are shown as (5). The minimum bandwidth requirements of each $s_{i}$ must be satisfied to guarantee QoS in a frame.

(v) The satisfaction ratio constraints are shown as (6). The satisfaction $y_{i}$ of each $s s_{i}$ will be greater than the variable $y$.

3.3. Greedy Algorithm for QoS-Based Max-Min Fair Scheduling. Though the ILP solution can be used to obtain optimal solutions for small-sized problem, but if the network scale grows larger, it has large time and space consumption for large-sized network. The heuristics algorithm is needed for better running time in large-sized network. In the following, we designed a heuristic algorithm.

3.3.1. Heuristic Algorithm. The strategy of the proposed heuristic algorithm is smallest total bandwidth requirement first and then applying spectral reuse scheme to assign resource. After QoS of all SSs is guaranteed, the max-min fair scheduling scheme is used to enhance the overall throughput and satisfaction ratio. The proposed heuristic algorithm has four steps as follows.

Step 1 (allocate limited resources to meet the QoS requirements of each SS). At first, the total number of tile requirements $\left(\operatorname{tr}_{i} \times \mathrm{hp}_{i}\right)$ of each $s_{i}$ is estimated. Then, the resources are allocated to all SSs by Algorithm 2 QoS_Scheduling() in increasing order of total tile requirements. Until the resources are not enough allocated or the requirements of all SSs are met, the scheduler will terminate the process of QoS_Scheduling().

Step 2 (increase the number of meeting QoS requirements). If the bandwidth requirements of $s_{i}$ are not satisfied, the spectral reuse mechanism is used to find available resource for each unmet SS in Algorithm 3 QoS_Spectral_Reuse(). The strategy of spectral reuse is to gain tiles from all allocated tiles of $s_{j}$; there is no link between each $s_{j}$ and the picked $s_{i}$. Hence, the satisfaction rate has an opportunity to increase. 


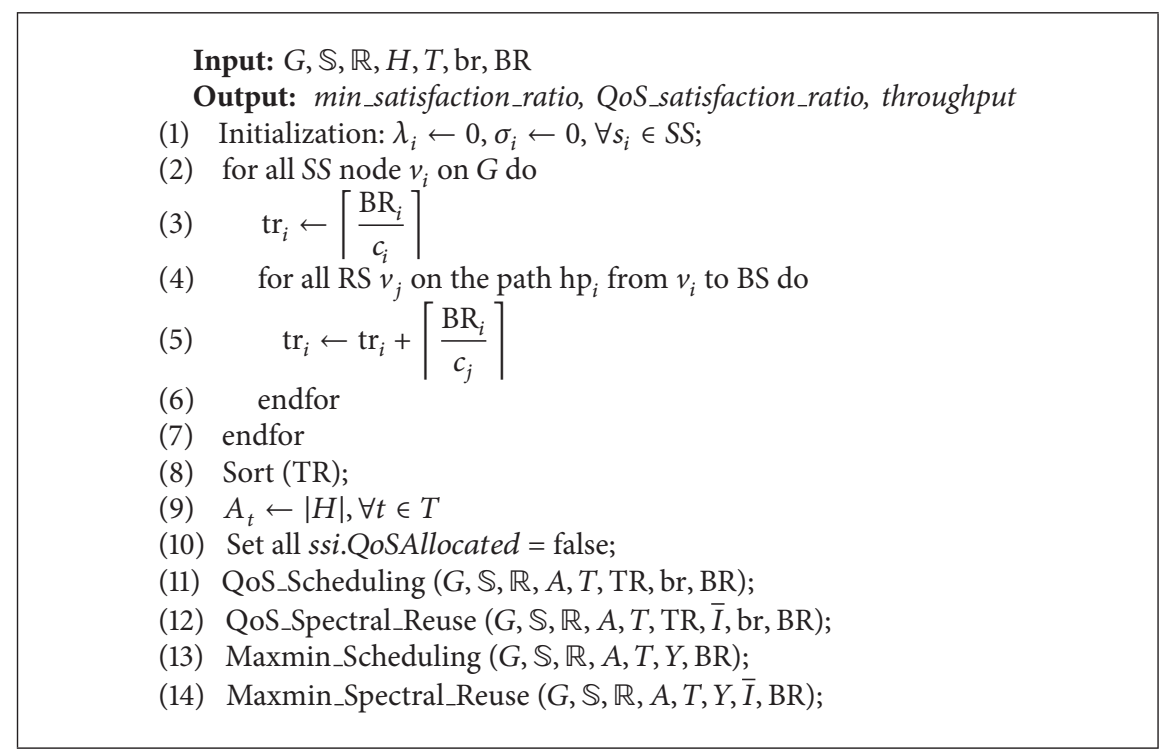

Algorithm 1: Heuristic Algorithm.

Output: $Y, A$

(1) Step 1. Choose a $s_{i}$ from SS set with the minimal total tile requirement $\operatorname{tr}_{i}$

(2) Step 2.

(3) index $\leftarrow 1$

(4) for $k=1 \rightarrow\left|\mathrm{hp}_{i}\right|$

(5) $\quad r e q \leftarrow\left\lceil\frac{\mathrm{br}_{i}}{c_{k}}\right\rceil$

(6) for $t=$ index $\rightarrow|T|$

(7) if $A_{t} \geq$ req

(8) $\quad A_{t} \leftarrow A_{t}-$ req; req $\leftarrow 0 ; k \leftarrow k+1$

(9) $\quad$ index $\leftarrow t ; t \leftarrow|T|$

(10) else

(11) $\quad$ if $(|T|-t)<\left(\mathrm{hp}_{i}-k\right)$

(12) free all tiles which allocated to $s_{i}$

(13) $\quad S S \leftarrow S S \backslash\left\{s_{i}\right\}$

(14) go to Step 1

(15) else

(16) $\quad A_{t} \leftarrow 0$; req $\leftarrow$ req $-A_{t}$; index $\leftarrow t ; t \leftarrow|T|$

(17) Step 3. if all hops of $s_{i}$ are allocated successfully

(18) $\mathbb{S} \leftarrow \mathbb{S} \backslash\left\{s_{i}\right\}$

(19) $\sigma_{i} \leftarrow\left\lceil\frac{\mathrm{br}_{i}}{c_{i}}\right\rceil ; \quad \lambda_{i} \leftarrow \sigma_{i} \times c_{i}$;

(20) for all RS $r s_{j}$ on the path from $s_{i}$ to BS do

(21) $\quad \sigma_{j} \leftarrow\left\lceil\frac{\mathrm{br}_{i}}{c_{j}}\right\rceil ; \quad \lambda_{j} \leftarrow \sigma_{j} \times c_{j}$;

(22) endfor

(23) $\quad y_{i} \leftarrow \frac{\lambda_{i}+\sum_{\forall r s_{j} \in \mathrm{hp}_{i}} \lambda_{j}}{\mathrm{BR}_{i} \times\left|\mathrm{hp}_{i}\right|}$;

(24) Step 4. if all SSs meet requirement or no sufficient resources can be allocated

(26) terminate scheduling else go to Step 1

Algorithm 2: QoS_Scheduling $(G, \mathbb{S}, \mathbb{R}, A, T$, TR, br, BR). 


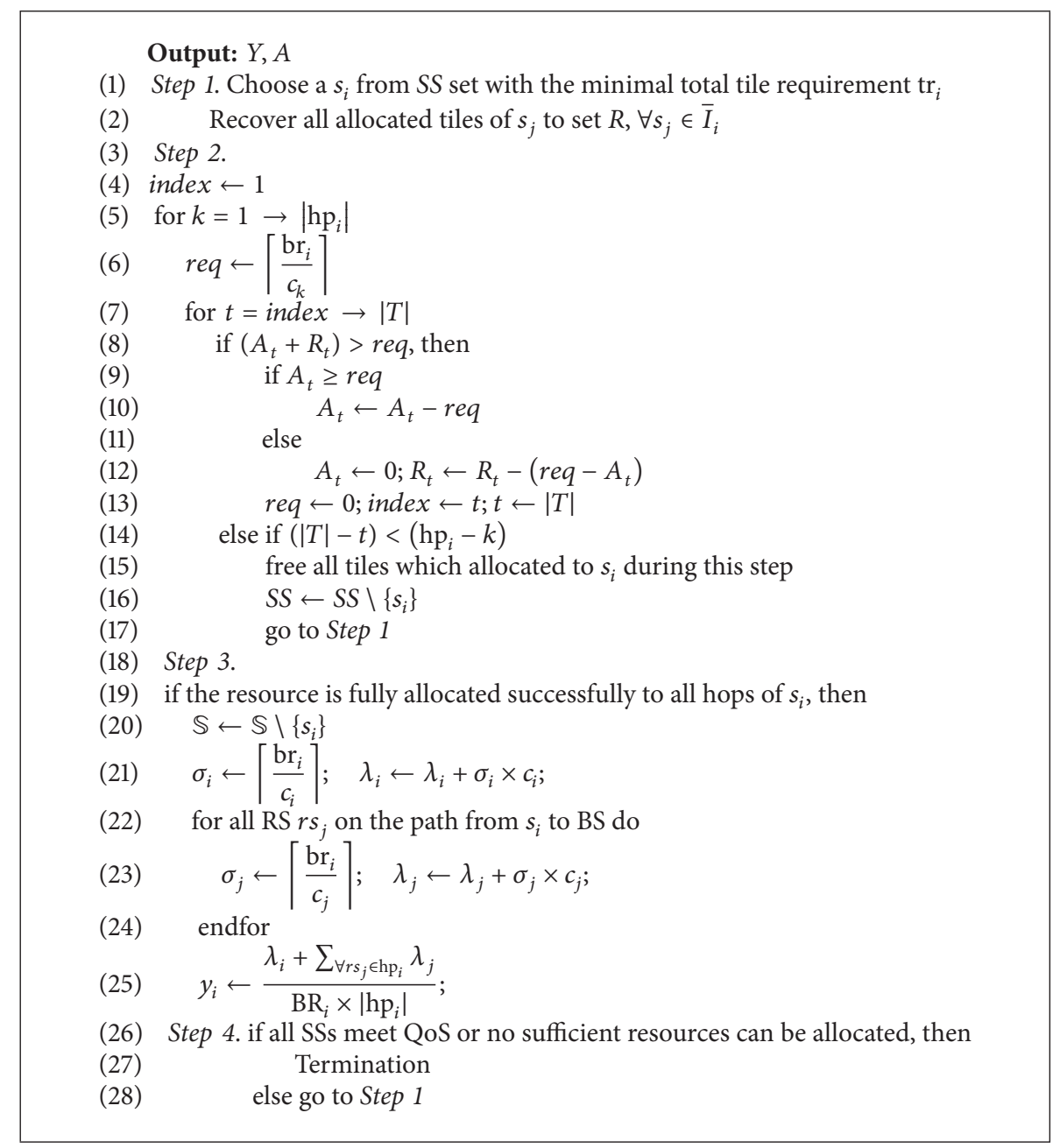

Algorithm 3: QoS_Spectral_Reuse $(G, \mathbb{S}, \mathbb{R}, A, T, \mathrm{TR}, \bar{I}$, br, BR).

Step 3 (remaining resources to do the max-min fair scheduling). When the first two steps are finished, then some remaining resources are available. Then, the available tiles are allocated to the SSs that have lowest satisfaction, and satisfaction ratio is upgraded by Algorithm 4 Maxmin_Scheduling().

Step 4 (upgrade the min satisfaction ratio). Finally, Algorithm 5 Maxmin_Spectral_Reuse() finds out an $s_{i}$ which has lowest satisfaction ratio among all SSs to increase its satisfaction ratio. Until all the SSs are allocated, available tiles by the spectral reuse mechanism or the satisfaction ratio of $s_{i}$ are equal to 1 . Then, the procedure of scheduling algorithm will be finished.

3.3.2. Scheduling Algorithm Description with an Example. As shown in Figure 1, the networks are composed of one BS, three RSs $=\left\{r_{1}, r_{2}, r_{3}\right\}$, and six SSs $=\left\{s_{1}, s_{2}, s_{3}, s_{4}, s_{5}, s_{6}\right\}$. The number of maximum bandwidth requirements of each SSs $\mathrm{BR}_{i}$ are $\{10,10,8,8,8$, and 10$\}$. The bandwidth requirements of SSs br $_{i}$ are $\{7,2,4,5,6$, and 3$\}$ for guaranteeing QoS. The capacity of each link $c_{l}$ is set to 1 . The number of time-slots $t$ is set to 7 . The number of subchannels $h$ is set to 3 . The interference of each RS and SS is defined as follows:

$$
\begin{array}{cc}
I\left(r_{1}\right)=\left\{s_{1}, s_{2}, r_{2}, r_{3}\right\}, & I\left(r_{2}\right)=\left\{r_{1}, r_{3}, s_{3}, s_{4}\right\}, \\
I\left(r_{3}\right)=\left\{r_{2}, s_{5}, s_{6}\right\}, \quad I\left(s_{1}\right)=\left\{s_{2}, r_{1}\right\}, \quad I\left(s_{6}\right)=\left\{s_{5}, r_{3}\right\} \\
I\left(s_{2}\right)=\left\{s_{1}, r_{1}, s_{3}\right\}, & I\left(s_{3}\right)=\left\{s_{2}, r_{4}, s_{4}\right\}, \\
I\left(s_{4}\right)=\left\{s_{3}, r_{2}, s_{5}\right\}, & I\left(s_{5}\right)=\left\{s_{4}, r_{3}, s_{6}\right\} .
\end{array}
$$

At first, the value of $\operatorname{tr}_{i}\left(=\left\lceil\mathrm{BR}_{i} / c_{i}\right\rceil+\sum_{\forall j \in \mathrm{hp}_{i} \backslash\left\{s_{i}\right\}}\left\lceil\mathrm{BR}_{i} / c_{j}\right\rceil\right)$ of each $s_{i}$ is calculated at lines (2)-(7) in Algorithm 1. Then, the value of TR is sorted by increasing order at line (8) in Algorithm 1. The value of parameter $A_{t}$ is initialized as $|H|$ for all time-slot $t$.

The limited resources are allocated by Algorithm 2. The $s_{i}$ is selected with minimum total tile requirement $\operatorname{tr}_{i}$ at line (1) of Algorithm 2. Then, the resources are allocated at lines (2)-(16) of Algorithm 2. After resources allocation are completed, the parameters and variables of the SS and RS will be updated at lines (17)-(23) of Algorithm 2. At lines 


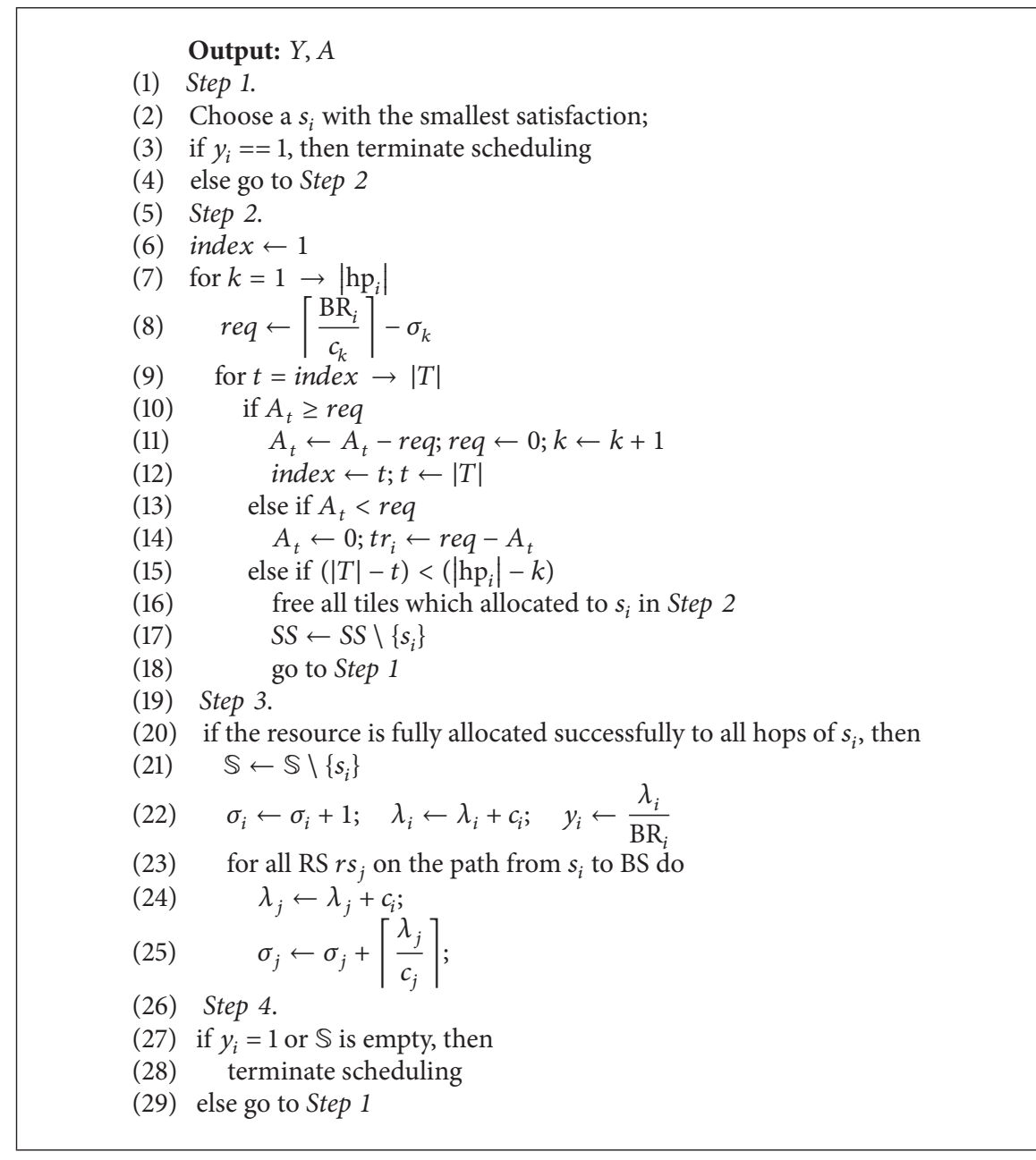

Algorithm 4: Maxmin_Scheduling $(G, \mathbb{S}, \mathbb{R}, A, T, Y, \mathrm{BR})$.

(24)-(26) of Algorithm 2 if no available resources are able to allocate to each SS, the procedure goes back to the main algorithm. Otherwise, the scheduler continue to find the next SS which can allocate resources to meet the QoS requirements of the SS. In our example, the total number of tiles is 21 . In Algorithm 2, the sequence of $s_{i}$ will be selected as $\left\{s_{2}, s_{6}, s_{3}\right\}$. The value of parameter $\operatorname{tr}_{i}$ is estimated as follows.

$$
\begin{aligned}
& \operatorname{tr}_{1}=\left\lceil\frac{\mathrm{BR}_{1}}{c_{1}}\right\rceil+\sum_{\forall j \in \mathrm{hp}_{1} \backslash\left\{s_{1}\right\}}\left\lceil\frac{\mathrm{BR}_{1}}{c_{j}}\right\rceil=14, \\
& \operatorname{tr}_{2}=\left\lceil\frac{\mathrm{BR}_{2}}{c_{2}}\right\rceil+\sum_{\forall j \in \mathrm{hp}_{2} \backslash\left\{s_{2}\right\}}\left\lceil\frac{\mathrm{BR}_{2}}{c_{j}}\right\rceil=4, \\
& \operatorname{tr}_{3}=\left\lceil\frac{\mathrm{BR}_{3}}{c_{3}}\right\rceil+\sum_{\forall j \in \mathrm{hp}_{3} \backslash\left\{s_{3}\right\}}\left\lceil\frac{\mathrm{BR}_{3}}{c_{j}}\right\rceil=8, \\
& \operatorname{tr}_{4}=\left\lceil\frac{\mathrm{BR}_{4}}{c_{4}}\right\rceil+\sum_{\forall j \in \mathrm{hp}_{4} \backslash\left\{s_{4}\right\}}\left\lceil\frac{\mathrm{BR}_{4}}{c_{j}}\right\rceil=10,
\end{aligned}
$$

$$
\begin{aligned}
& \operatorname{tr}_{5}=\left\lceil\frac{\mathrm{BR}_{5}}{c_{5}}\right\rceil+\sum_{\forall j \in \mathrm{hp}_{5} \backslash\left\{s_{5}\right\}}\left\lceil\frac{\mathrm{BR}_{5}}{c_{j}}\right\rceil=12, \\
& \operatorname{tr}_{6}=\left\lceil\frac{\mathrm{BR}_{6}}{c_{6}}\right\rceil+\sum_{\forall j \in \mathrm{hp}_{6} \backslash\left\{s_{6}\right\}}\left\lceil\frac{\mathrm{BR}_{6}}{c_{j}}\right\rceil=6 .
\end{aligned}
$$

The scheduling results of Algorithm 2 are shown in Figure 2.

Moreover, if the bandwidth requirements of some SSs are still unsatisfied, the number of SS with QoS guarantee will be raised by Algorithm 3. The bandwidth requirement unsatisfied $s_{i}$ will be found with the minimum $\operatorname{tr}_{i}$ at line (1) of Algorithm 3. All allocated tiles of $s_{j}, \forall s_{j} \in \bar{I}_{i}$, are recovered to set $R$ at line (2) of Algorithm 3. Then, the spectral reuse strategy is used to maximize satisfaction ratio at lines (3)-(17) in Algorithm 3 for picked $s_{i}$. If the available tiles $A_{t}+R_{t}$ can meet the requirement of $s_{i}$ at time slot $t$ for $k$ th hop, the required tiles are allocated at lines (8)-(13) of Algorithm 3. While the requirements of all hops are met, the parameters and variables of the SS and RS will be updated at 


\section{Output: $Y$}

(1) Step 1. Choose a $s_{i}$ with the smallest satisfaction;

(2) if $y_{i}=1$, then terminate scheduling

(3) else Recover all allocated tiles of $s_{j}$ to set $R, \forall s_{j} \in \overline{I_{i}}$

(4) go to Step 2

(5) Step 2.

(6) index $\leftarrow 1$

(7) for $k=1 \rightarrow \mathrm{hp}_{i}$

(8) $\quad r e q \leftarrow \operatorname{tr}_{i}$

(9) for $t=$ index $\rightarrow|T|$

(10) if $A_{t} \geq$ req, then

(11) $\quad A_{t} \leftarrow A_{t}-$ req; req $\leftarrow 0 ; k \leftarrow k+1$

(12) $\quad$ index $\leftarrow t ; t \leftarrow|T|$

(13) else

(16)

(19)

(20)

(22) Step 3. if all hops of $s_{i}$ are fully allocated success

(23) $\quad \sigma_{i} \leftarrow \sigma_{i}+1 ; \quad \lambda_{i} \leftarrow \lambda_{i}+c_{i} ; \quad y_{i} \leftarrow \frac{\lambda_{i}}{\mathrm{BR}_{i}} ;$

(24) for all RS $r s_{j}$ on the path from $s_{i}$ to BS do

(25) $\quad \lambda_{j} \leftarrow \lambda_{j}+c_{i}$;

(27) end for

$$
\begin{aligned}
& \lambda_{j} \leftarrow \lambda_{j}+c_{i} ; \\
& \sigma_{j} \leftarrow \sigma_{j}+\left\lceil\frac{\lambda_{j}}{c_{j}}\right\rceil ;
\end{aligned}
$$

(28) Step 4.

(29) if $y_{i}=1$ or $\mathbb{S}$ is empty, then

(30) terminate scheduling

(31) else go to Step 1

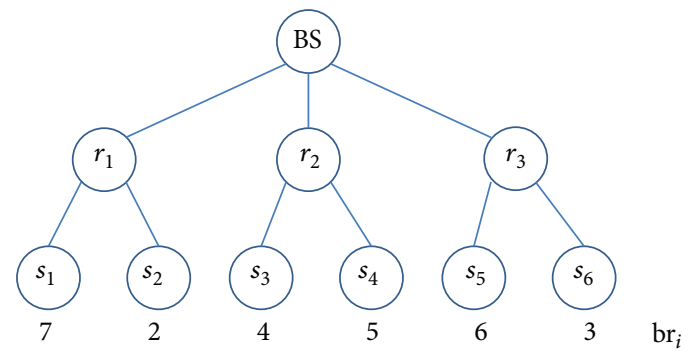

OFDMA frame:

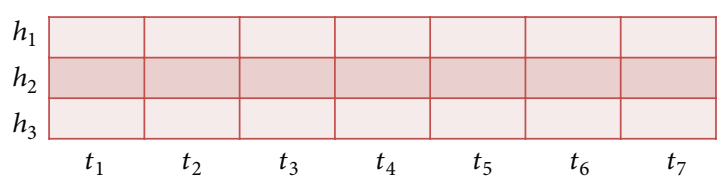

Figure 1: The network topology. 


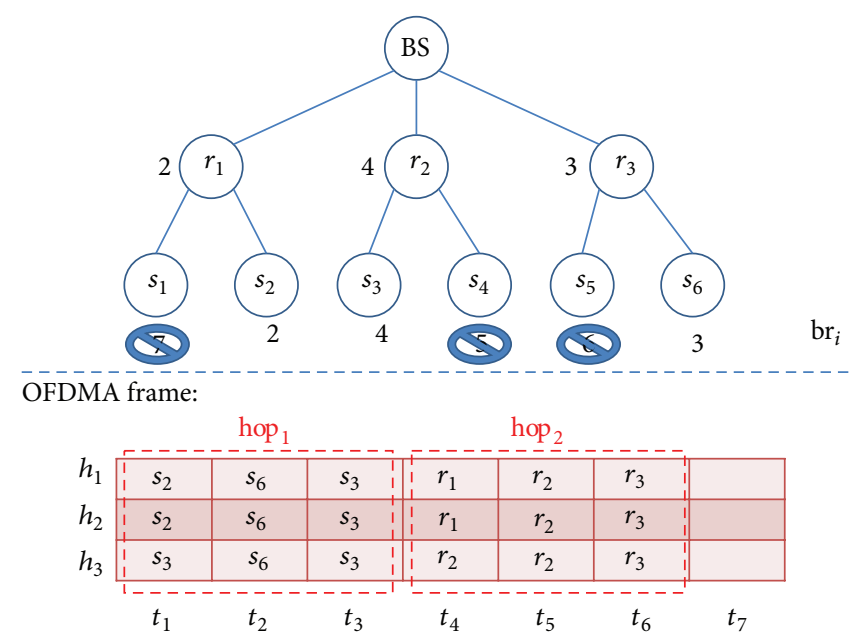

FIGURE 2: The allocation of resources meets the QoS requirements of $s_{2}, s_{3}$, and $s_{6}$.

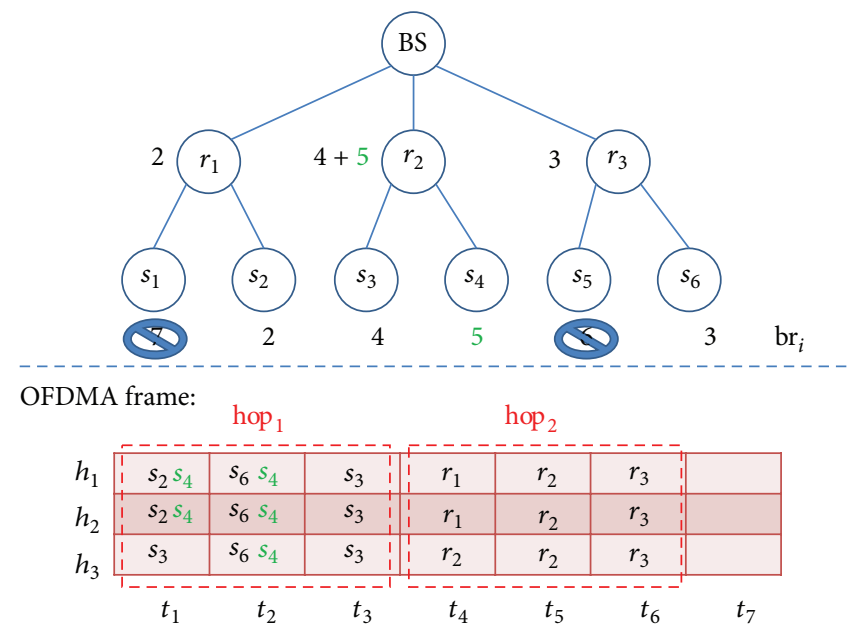

FIGURE 3: $s_{4}$ performs the spectral reuse.

lines (18)-(25) in Algorithm 3. Otherwise, all acquired tiles of $s_{i}$ are freed at line (15) of Algorithm 3. If the resources allocation have not been finished, the scheduler will find out the next one to meet the bandwidth requirements of $s_{i}$ to guarantee QoS by the spectral reuse strategy. Until the minimum bandwidth requirements of all SSs are satisfied or the available tiles are not enough to allocate, then this step will be terminated. The results of first hop of $s_{4}$ are scheduled by spectral reuse strategy of Algorithm 3 as shown in Figure 3. Because the available tiles are insufficient at second hop, $s_{4}$ cannot be assigned as shown in Figure 4. Hence, all acquired tiles of $s_{4}$ need to be freed. Due to the available tiles which are not enough to assign, this step is terminated.

If the remaining available tiles have not been allocated completely, then these remaining resources can increase the network minimum satisfaction ratio by Algorithm 4. The SS would be picked with lowest satisfaction ratio in sequence at line (2) of Algorithm 4. While the lowest satisfaction ratio is equal to 1 , the scheduling of Algorithm 4 is terminated.
Otherwise, the scheduler will check whether there available tiles can be assigned to the $s_{i}$ at lines (5)-(18) of Algorithm 4, if the resources could be allocated to the SS. After the parameters and variables of SS and RS are updated at lines (19)-(26) of Algorithm 4, the scheduler continue to find out the lowest satisfaction ratio of SS and to assign available tiles to increase satisfaction ratio. Until no available tiles can be allocated or the requirements of all SSs are met, the scheduling procedure is terminated. The max-min fair scheduling is performed with remaining tiles in Algorithm 4. The satisfaction ratio of $\left\{s_{1}, s_{4}, s_{5}\right\}$ is 0 . Because the second hop has no enough available tiles, the schedule fails for $\left\{s_{1}, s_{4}, s_{5}\right\}$. The scheduling results of Algorithm 4 are shown in Figure 5.

Finally, the spectral reuse strategy is operated on the SS which has lowest satisfaction ratio for increasing the minimum satisfaction ratio in Algorithm 5. At line (1) of Algorithm 5, the scheduler pick a $s_{i}$ that has lowest satisfaction ratio. If the lowest satisfaction ratio equals to 1 at line (2) 


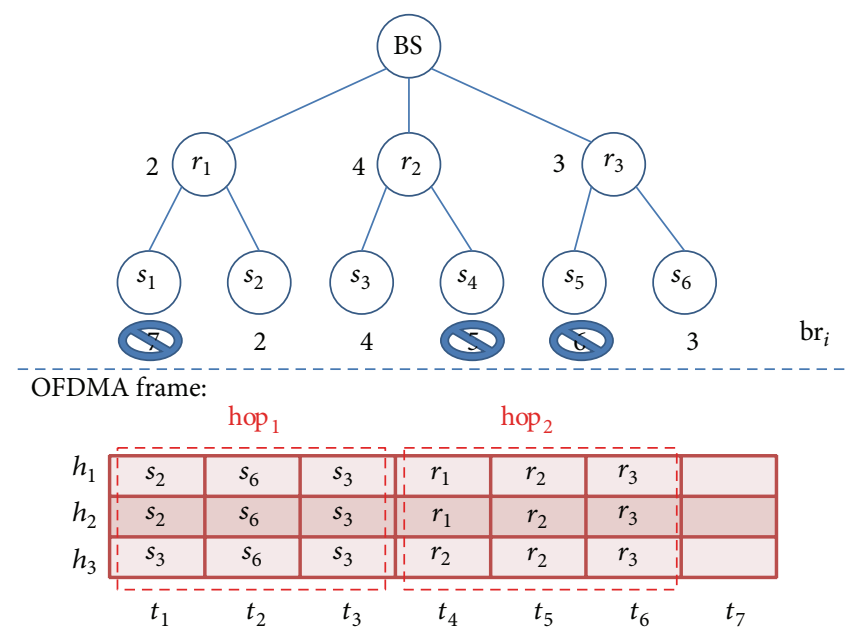

FIGURE 4: When lacking resources, $s_{4}$ cannot perform spectral reuse.

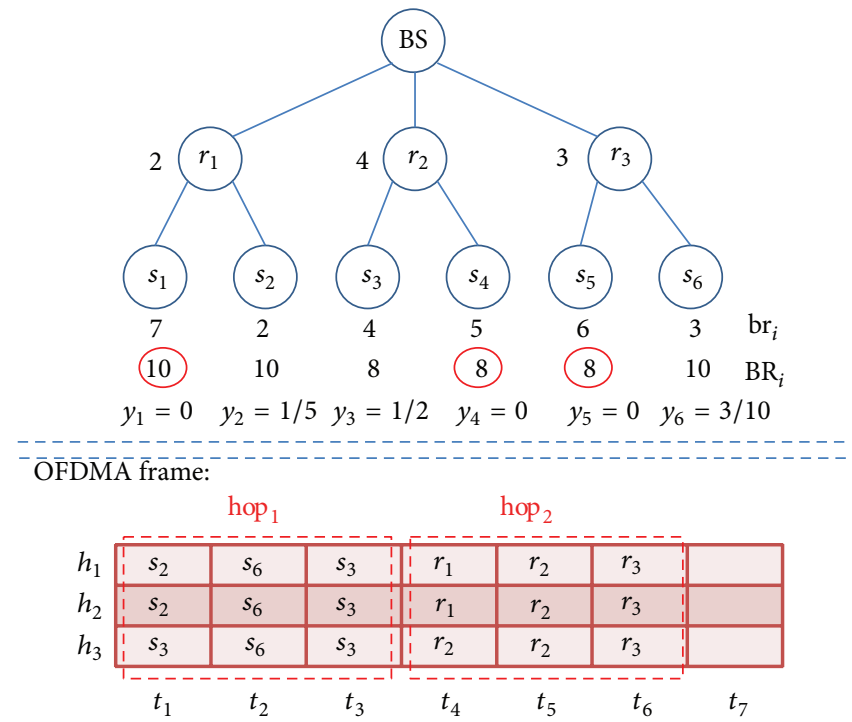

FIGURE 5: No remaining resources can be used to perform max-min fair scheduling.

of Algorithm 5, the scheduling of Algorithm 5 is terminated. Otherwise, all allocated tiles of $s_{j}$ are recovered to set $R, \forall s_{j} \in$ $\bar{I}_{i}$ at line (3) of Algorithm 5. Then, the spectral reuse strategy is used to allocate tiles to $s_{i}$ for maximizing satisfaction ratio at lines (5)-(21) of Algorithm 5. When the available tiles set $A_{t}$ enough to assign to $k$ th hop, the tiles of $A_{t}$ set are firstly allocated at lines (10)-(12) of Algorithm 5. When the set $A_{t}$ cannot fulfill the req of $s_{i}$, both the set $A_{t}$ and $R_{t}$ are applied to allocate at lines (13)-(21) of Algorithm 5. When both the set $A_{t}$ and $R_{t}$ cannot satisfy the req of $s_{i}$ at time slot $t$ at line (21) of Algorithm 5, the difference of required tiles would be found at next time slot. If the requirement of all hops cannot be met, all acquired tiles of $s_{i}$ have to be freed at lines (16)-(19) of Algorithm 5. Then, the scheduler continue to find out next SS until no resources can be allocated. By Algorithm 4, the scheduled satisfaction ratio of $\left\{s_{1}, s_{4}, s_{5}\right\}$ is
0 . Algorithm 5 enhances satisfaction ratio of $\left\{s_{1}, s_{4}, s_{5}\right\}$ to $\{0.1,0.125,0.125\}$. The scheduling results of Algorithm 5 are shown in Figure 6.

Finally, we get min satisfaction ratio $=0.1$, QoS satisfaction ratio $=0.5$, and throughput $=12$, as shown in Figure 7 .

\section{Simulation}

In this section, we implement our heuristic algorithms and the algorithm proposed in [21] for performance comparison. We compare three parameters in the experimental results, namely, average minimum satisfaction ratio, average QoS satisfaction ratio, and average throughput.

4.1. Environmental Setup. For experimental environment setting, SS transmission range and interference range are 


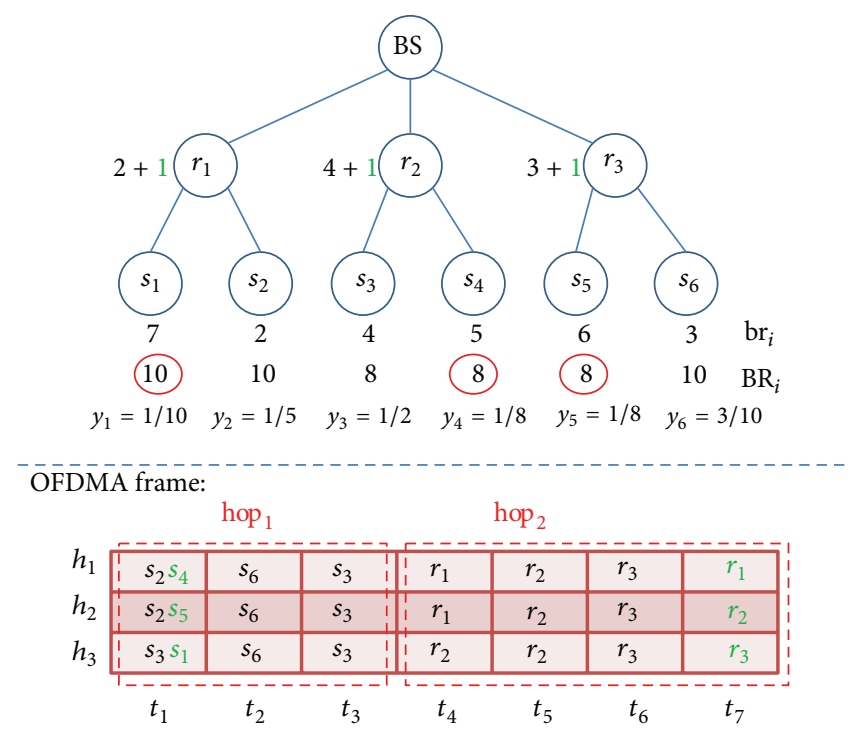

FIGURE 6: Using spectral reuse to perform max-min fair scheduling.

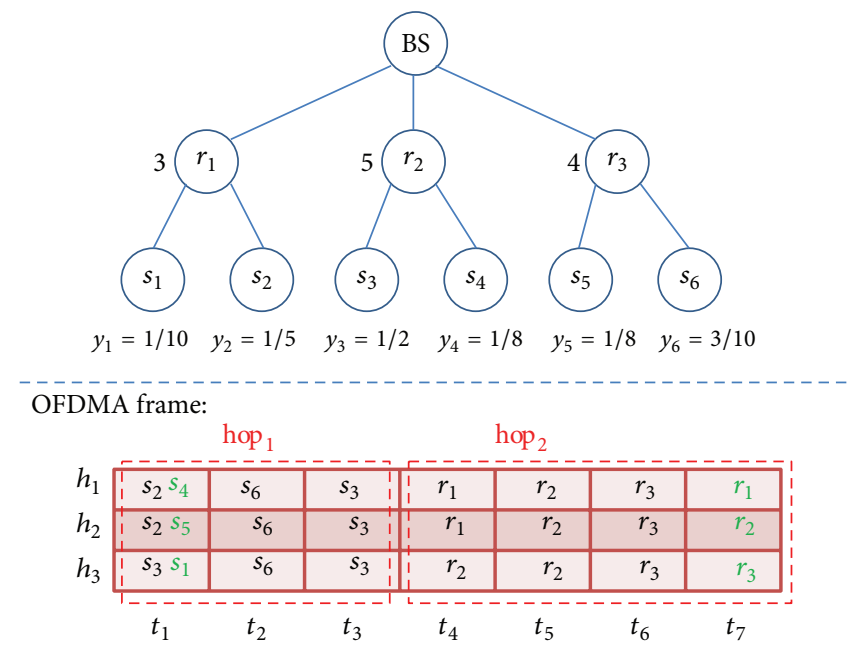

Figure 7: The result of example.

set 1000 and 1000; RS and the BS transmission range and interference range are set 1000 and 2000. BS was deployed at the center of the field. Multihop shortest path routing was adopted to obtain the network topology. SS is distributed in the field by random, each data packet requirement and QoS requirement of SS are set randomly among 2 to 8, and the QoS requirements of each SS must be less than or equal to the data packet requirement.

In the beginning, we use our heuristic algorithm and scheduling method of [21] in $1500 \times 1500$ square units and deployed 4 RSs to compare with three goals. These three goals are minimum satisfaction ratio, QoS satisfaction ratio, and throughput. For OFDMA setting, the number of time slots and subchannels are 12 and 5 in a frame. Then, we consider another large scale network which has $3000 \times 3000$ square units and deploy 16 RSs. For OFDMA setting, time-slots and subchannels number are 48 and 5 in a frame.
4.2. Experiment Results. Then we compare heuristic method with [21] on the experimental results of these two methods by three goals. The three goals are the ratio of average minimum satisfaction, average QoS satisfaction ratio, and average throughput.

When the number of SSs is increasing in Figure 8(a), it will lead to insufficient resources to allocate for all SSs then make min satisfaction ratio decrease. Our method allocates the resources to the proposed QoS requirements of SS at first priority and does the max-min fair allocation scheduling on the remaining resources. We find that the method of [21] is better than our method in terms of average min satisfaction ratio.

When the field changed to $3000 \times 3000$, then 16 RSs are placed for experiment, and the number of SSs increased from 10 to 100 . We can find that the number of SSs increased and 


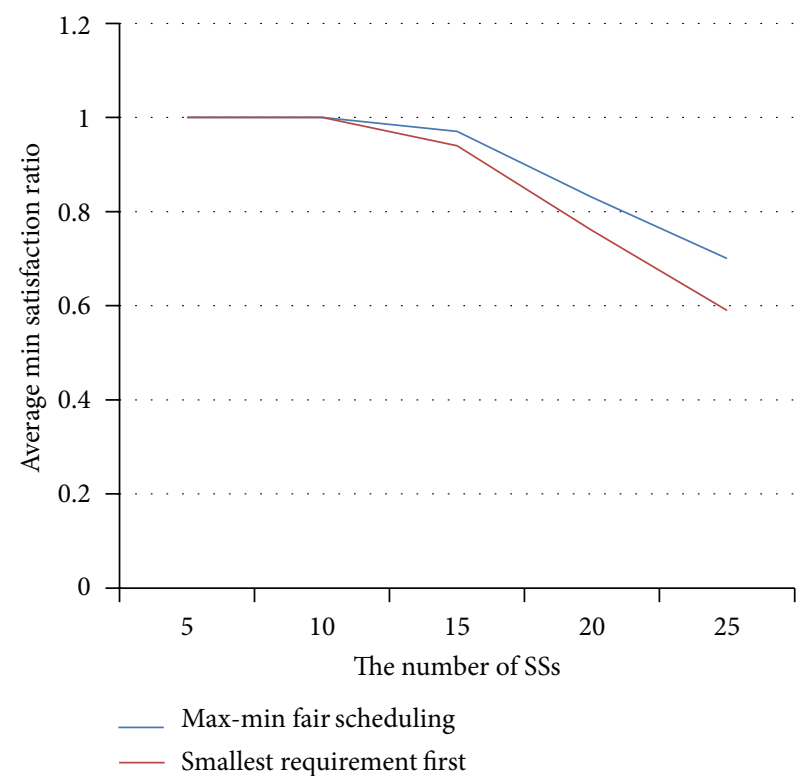

(a) Field: $1500 \times 1500,4$ RS

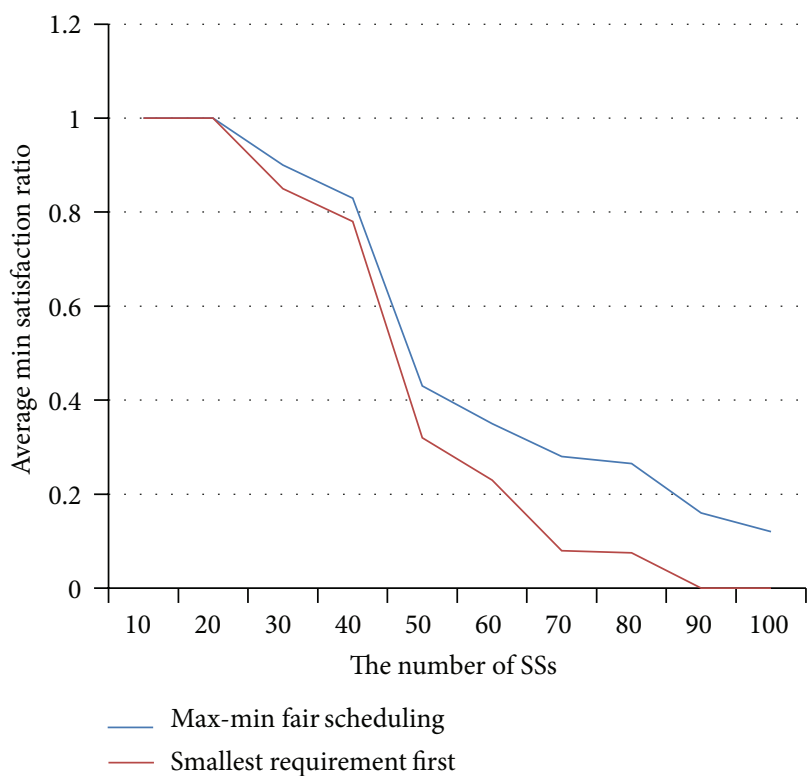

(b) Field: $3000 \times 3000,16$ RS

FIGURE 8: Average min satisfaction ratio comparison.

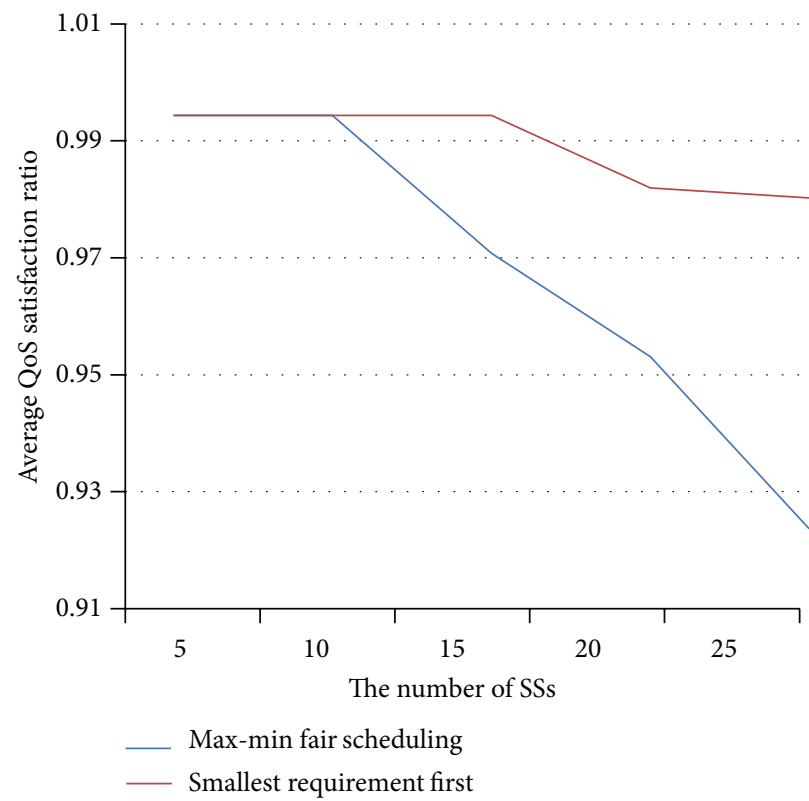

(a) Field: $1500 \times 1500,4$ RS

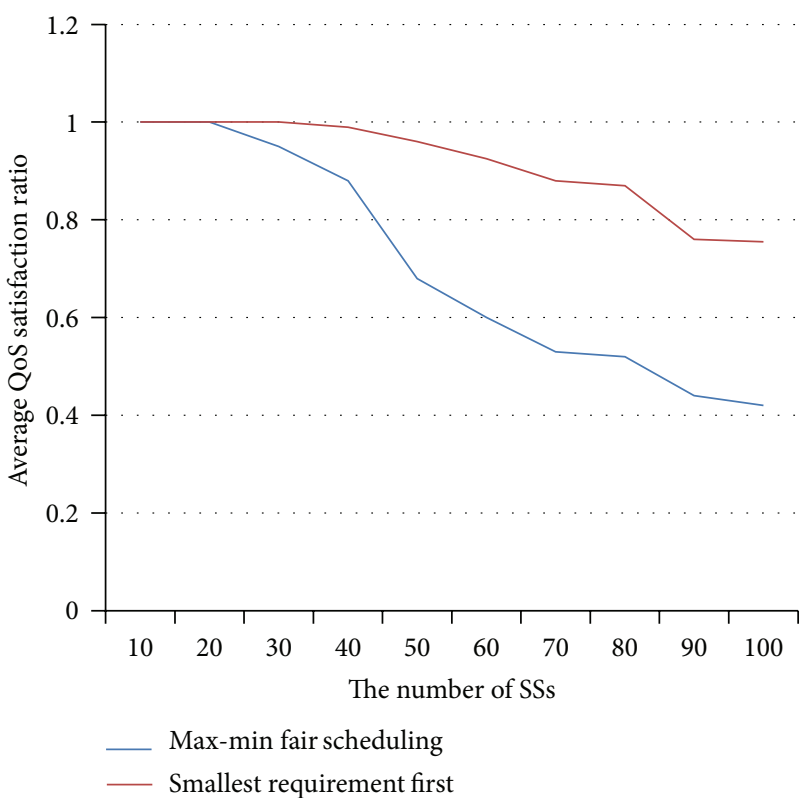

(b) Field: $3000 \times 3000,16$ RS

FIgURE 9: Average QoS satisfaction ratio comparison.

the number of hops increased in Figure 8(b), then the overall average min satisfaction ratio will decline obviously.

In Figure 9, our heuristic algorithm will be higher than the method of [21] when comparing with average QoS satisfaction ratio. In order to arrange the QoS requirements in increasing order and allocate resources in increasing order, our heuristic algorithm is better than [21] in terms of QoS satisfaction ratio.

From Figure 10, we can find that the average throughput of the two methods is almost equal. When the number of hops increase, the average throughput of two methods is also almost equal.

\section{Conclusion}

In this paper, we study the multihop fairness scheduling problem with QoS control for enhancing throughput and guaranteeing QoS in WiMAX mesh networks. For allocating resource to multiple SSs, fairness is a key concern. The notion of max-min fairness is applied as our metric to define the 


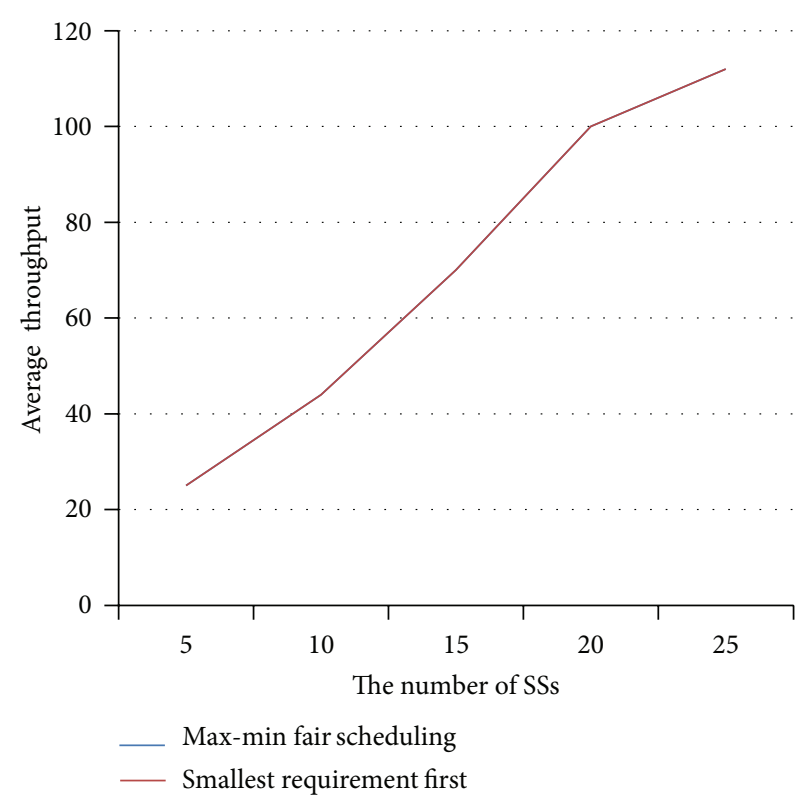

(a) Field: $1500 \times 1500,4$ RS

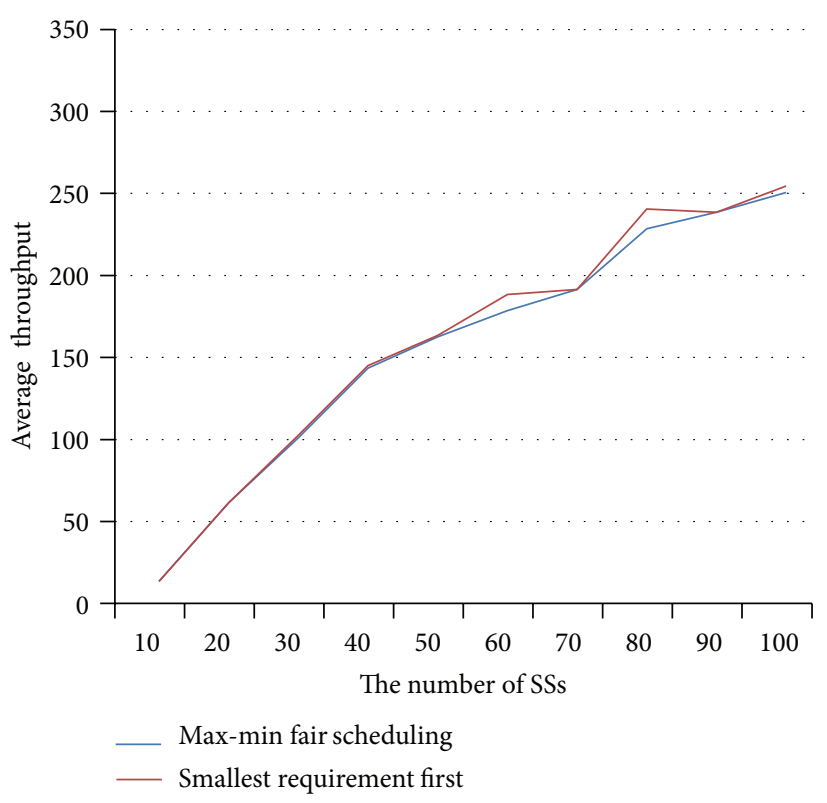

(b) Field: $3000 \times 3000,16$ RS

FIgURE 10: Average network throughput comparison.

QoS-based max-min fair scheduling problem for maximizing the minimum satisfaction ratio of each SS. We formulate an integer linear programming (ILP) model to provide an optimal solution on small-scale networks. Although the ILP solution can be used to obtain optimal solutions for smallscale network, it has high operation time and space consumption for large-scale networks.

Therefore, in the paper, several heuristic scheduling algorithms are proposed to maximize both the minimum satisfaction ratio and the QoS satisfaction ratio based on Orthogonal Frequency-Division Multiple Access (OFDMA) model in the networks. The strategy of proposed heuristic algorithm is smallest total bandwidth requirement first and then applying spectral reuse scheme to assign resource. After QoS of all SSs is guaranteed, the max-min fair scheduling scheme is used to enhance the overall throughput and satisfaction ratio. Experimental results show that our method is better than previous work in terms of QoS satisfaction ratio and throughput.

\section{Acknowledgments}

This work was supported in part by Taiwan NSC under Grant nos. NSC 102-2221-E-274-004 and NSC 102-2221-E-194-054. The authors would like to thank the reviewers for their insightful comments which helped to significantly improve the paper.

\section{References}

[1] "IEEE 802.16e-2006 Standard, Part 16: Air Interface for Fixed and Mobile Broadband Wireless Access Systems-Amendment for Physical and Medium Access Control Layers for Combined Fixed and Mobile Operation in Licensed Bands," IEEE, 2006.
[2] G. Li and H. Liu, "Downlink radio resource allocation for multi-cell OFDMA system," IEEE Transactions on Wireless Communications, vol. 5, no. 12, pp. 3451-3459, 2006.

[3] A. Belghith, L. Nuaymi, and P. Maillé, "Pricing of real-time applications in WiMAX systems," in Proceedings of the 68th Semi-Annual IEEE Vehicular Technology Conference (VTC '08), pp. 1-6, September 2008.

[4] I. G. Fraimis, V. D. Papoutsis, and S. A. Kotsopoulos, "A decentralized subchannel allocation scheme with Inter-cell Interference Coordination (ICIC) for multi-cell OFDMA systems," in Proceedings of the 53rd IEEE Global Communications Conference (GLOBECOM '10), December 2010.

[5] V. Chandrasekhar, J. G. Andrews, and A. Gatherer, "Femtocell networks: a survey," IEEE Communications Magazine, vol. 46, no. 9, pp. 59-67, 2008.

[6] Y. Kim and M. L. Sichitiu, "Fairness schemes in 802.16j mobile multihop relay networks," in Proceedings of the 53rd IEEE Global Communications Conference (GLOBECOM '10), December 2010.

[7] V. Gunasekaran and F. C. Harmantzis, "Affordable infrastructure for deploying WiMAX systems: Mesh v. Non Mesh," in Proceedings of the IEEE 61st Vehicular Technology Conference (VTC '05), vol. 5, pp. 2979-2983, June 2005.

[8] H. Shetiya and V. Sharma, "Algorithms for routing and centralized scheduling in IEEE 802.16 mesh networks," in Proceedings of the IEEE Wireless Communications and Networking Conference (WCNC '06), vol. 1, pp. 147-152, April 2006.

[9] C.-Y. Hong and A.-C. Pang, "Link scheduling with QoS guarantee for wireless relay networks," in Proceedings of the 28th Conference on Computer Communications IEEE (INFOCOM '09), pp. 2806-2810, April 2009.

[10] L. Fu, Z. Cao, and P. Fan, "Spatial reuse in IEEE 802.16 based wireless mesh networks," in Proceedings of the International Symposium on Communications and Information Technologies (ISCIT '05), pp. 1311-1314, October 2005. 
[11] L.-W. Chen, Y.-C. Tseng, Y.-C. Wang, D.-W. Wang, and J.-J. Wu, "Exploiting spectral reuse in routing, resource allocation, and scheduling for IEEE 802.16 mesh networks," IEEE Transactions on Vehicular Technology, vol. 58, no. 1, pp. 301-313, 2009.

[12] S. Ramanathan and E. L. Lloyd, "Scheduling algorithms for multihop radio networks," IEEE/ACM Transactions on Networking, vol. 1, no. 2, pp. 166-177, 1993.

[13] I. F. Akyildiz, X. Wang, and W. Wang, "Wireless mesh networks: a survey," Computer Networks, vol. 47, no. 4, pp. 445-487, 2005.

[14] G. Li and H. Liu, "On the capacity of broadband relay networks," in Proceedings of the Conference Record of the 38th Asilomar Conference on Signals, Systems and Computers, pp. 1318-1322, November 2004.

[15] M. K. Awad and X. Shen, "OFDMA based two-hop cooperative relay network resources allocation," in Proceedings of the IEEE International Conference on Communications (ICC '08), pp. 4414-4418, May 2008.

[16] H.-Y. Wei, S. Ganguly, R. Izmailov, and Z. J. Haas, "Interferenceaware IEEE 802.16 WiMax mesh networks," in Proceedings of the IEEE 61st Vehicular Technology Conference (VTC '05), vol. 5, pp. 3102-3106, June 2005.

[17] L. Tassiulas and S. Sarkar, "Maxmin fair scheduling in wireless networks," in Prceedings of the IEEE Infocom, pp. 763-772, June 2002.

[18] S. C. Liew and Y. J. Zhang, "Proportional fairness in multichannel multi-rate wireless networks," in Proceedings of the IEEE Global Telecommunications Conference (GLOBECOM '06), December 2006.

[19] A. Sayenko, O. Alanen, J. Karhula, and T. Hämäläinen, "Ensuring the QoS requirements in 802.16 scheduling," in Proceedings of the 9th ACM Symposium on Modeling, Analysis and Simulation of Wireless and Mobile Systems (ACM MSWiM'06), pp. 108117, October 2006.

[20] M. Andrews and L. Zhang, "Scheduling algorithms for multicarrier wireless data systems," in Proceedings of the 13th Annual ACM International Conference on Mobile Computing and Networking (MobiCom '07), pp. 3-14, September 2007.

[21] S. Bai, W. Zhang, Y. Liu, and C. Wang, "Max-min fair scheduling in OFDMA-based multi-hop WiMAX mesh networks," in Proceedings of the IEEE International Conference on Communications (ICC '11), June 2011.

[22] S. Deb, V. Mhatre, and V. Ramaiyan, "WiMAX relay networks: Opportunistic scheduling to exploit multiuser diversity and frequency selectivity," in Proceedings of the 14th Annual International Conference on Mobile Computing and Networking (MobiCom '08), pp. 163-174, September 2008.

[23] K. Sundaresan, W. Wang, and S. Eidenbenz, "Algorithmic aspects of communication in ad-hoc networks with smart antennas," in Proceedings of the 7th ACM International Symposium on Mobile Ad Hoc Networking and Computing (MOBIHOC '06), pp. 298-309, May 2006.

[24] Y. Xu, S. Wan, J. Tang, and R. S. Wolff, "Interference Aware routing and scheduling in wireless backhaul networks with smart antennas," in Proceedings of the 6th Annual IEEE Communications Society Conference on Sensor, Mesh and Ad Hoc Communications and Networks (SECON '09), June 2009. 


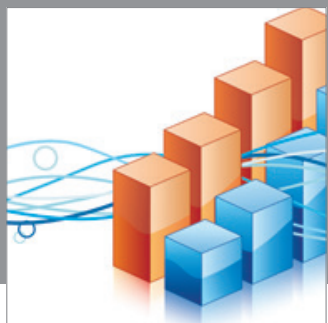

Advances in

Operations Research

mansans

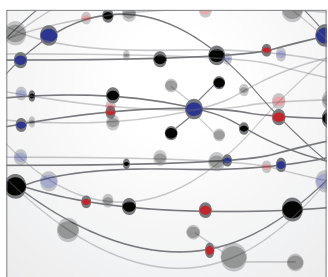

The Scientific World Journal
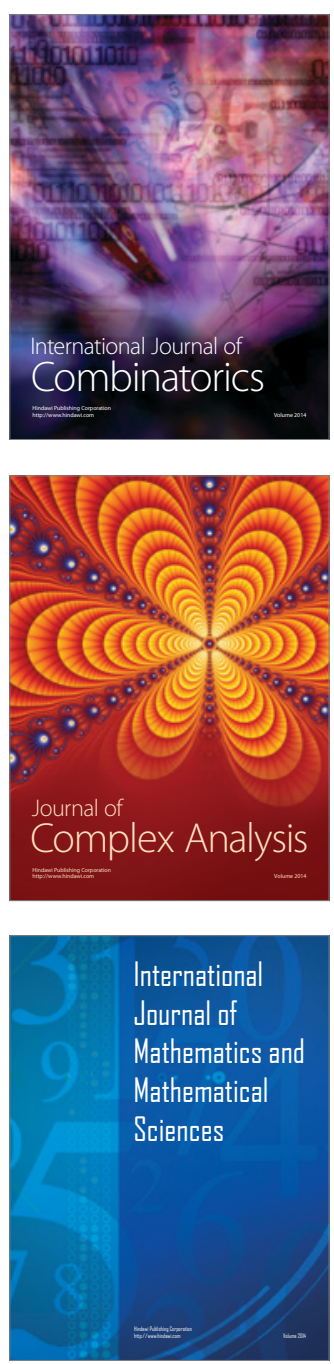
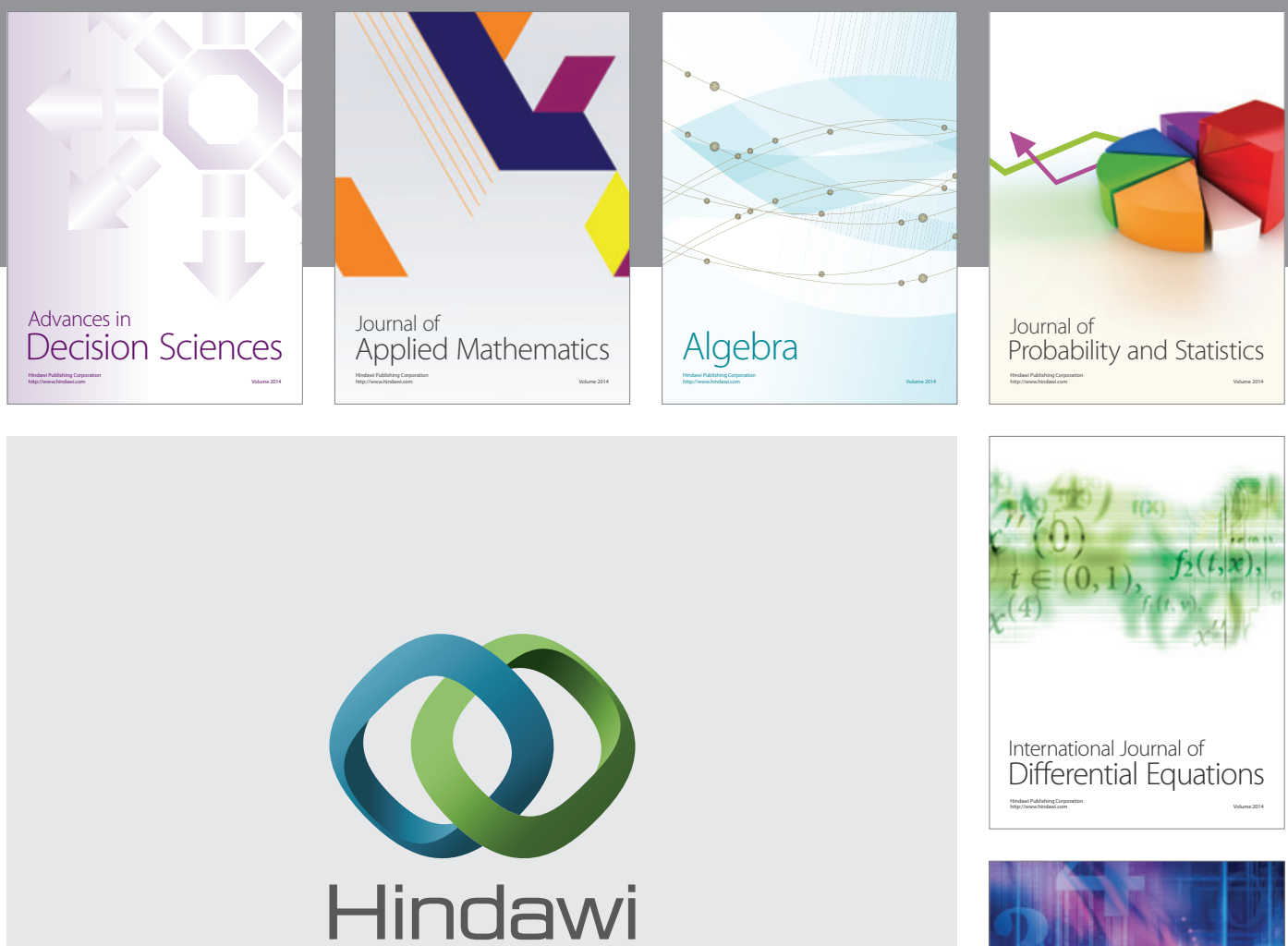

Submit your manuscripts at http://www.hindawi.com
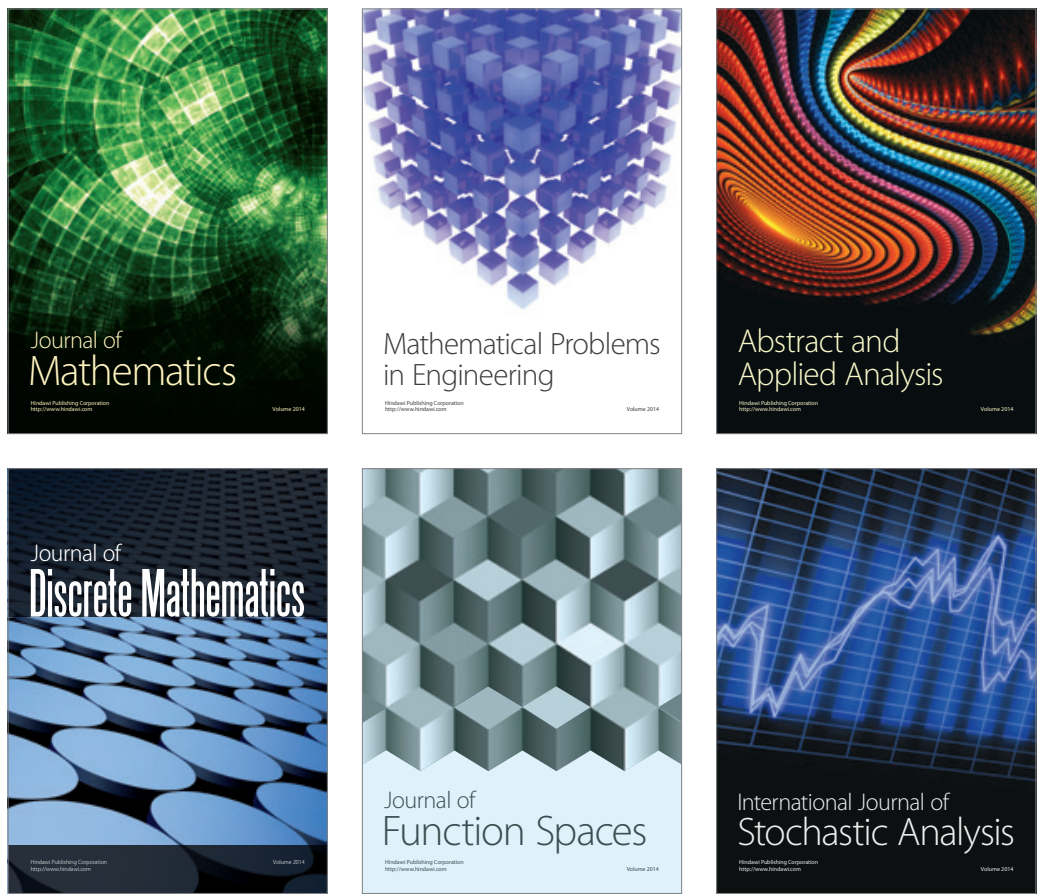

Journal of

Function Spaces

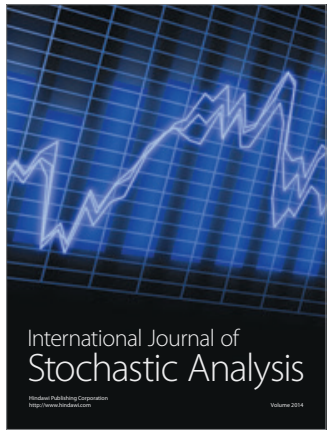

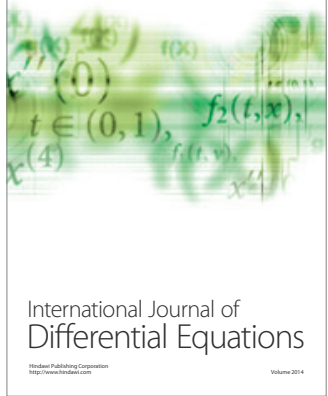
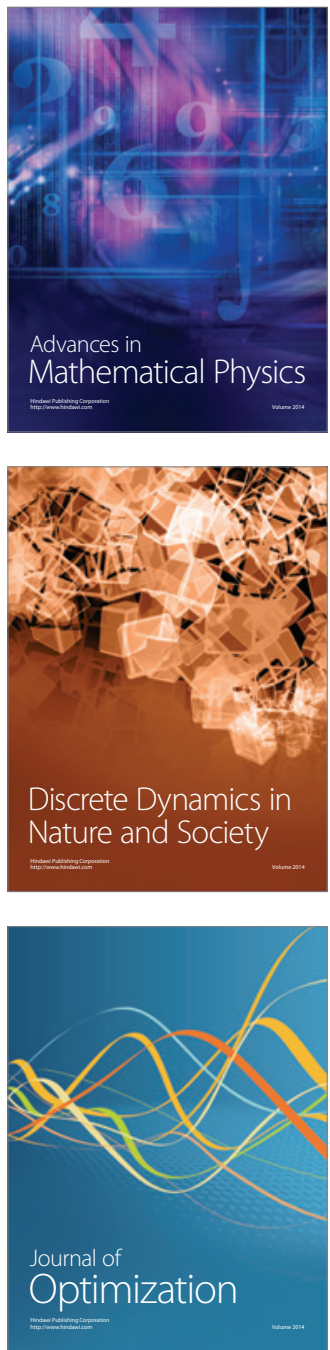